\title{
Properties of Subtree-Prune-and-Regraft Operations on Totally-Ordered Phylogenetic Trees
}

\author{
Yun S. Song ${ }^{\star \star}$ \\ Department of Statistics, University of Oxford, 1 South Parks Road, Oxford, OX1 3TG, UK \\ song@stats.ox.ac.uk
}

The date of receipt and acceptance will be inserted by the editor

AMS Subject Classification: 05C05, 92D15

\begin{abstract}
We study some properties of subtree-prune-and-regraft (SPR) operations on leaflabelled rooted binary trees in which internal vertices are totally ordered. Since biological events occur with certain time ordering, sometimes such totally-ordered trees must be used to avoid possible contradictions in representing evolutionary histories of biological sequences. Compared to the case of plain leaf-labelled rooted binary trees where internal vertices are only partially ordered, SPR operations on totally-ordered trees are more constrained and therefore more difficult to study. In this paper, we investigate the unit-neighbourhood $U(T)$, defined as the set of totally-ordered trees one SPR operation away from a given totally-ordered tree $T$. We construct a recursion relation for $|U(T)|$ and thereby arrive at an efficient method of determining $|U(T)|$. In contrast to the case of plain rooted trees, where the unit-neighbourhood size grows quadratically with respect to the number $n$ of leaves, for totally-ordered trees $|U(T)|$ grows like $O\left(n^{3}\right)$. For some special topology types, we are able to obtain simple closed-form formulae for $|U(T)|$. Using these results, we find a sharp upper bound on $|U(T)|$ and conjecture a formula for a sharp lower bound. Lastly, we study the diameter of the space of totally-ordered trees measured using the induced SPR-metric.
\end{abstract}

Keywords: subtree prune regraft, ordered trees, neighbourhood, recombination

\section{Introduction}

Leaf-labelled binary trees, also known as binary phylogenetic trees, are widely used in representing evolutionary histories of biological sequences. Furthermore, some problems in evolutionary genetics naturally involve considering more than a single tree, and it therefore is important to have a method of comparing trees. Exactly how dissimilar-

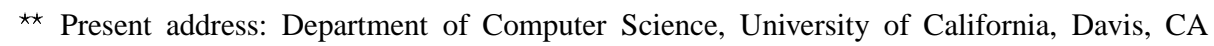
95616, USA. E-mail: yssong@ @s.ucdavis.edu 
ities between trees should be measured depends on the kind of trees being considered, as well as on the underlying problem.

A case in which one is inevitably led to use a collection of trees is the study of genealogies subject to recombination. Recombination can cause different regions in DNA sequences to have different evolutionary histories. In such a case, one is often interested in knowing how two trees differ in their topologies, the main idea being that a quantitative measure of the difference in tree topologies should reflect the number of detectable recombination events.

A type of tree rearrangement operation useful for studying topology changes due to recombination is the so-called subtree-prune-and-regraft (SPR) operation $[1,6]$. As the name indicates, an SPR operation roughly involves cutting an edge from a tree $T$, thus "pruning" a subtree $t$ from $T$, and then "regrafting" $t$ onto somewhere in the remaining part of $T$. One important point to note is that the precise defi nition of an SPR operation depends on the type of the tree on which the operation is being performed.

In [5], SPR operations on trees were used to address the problem of determining the minimum number of recombination events while constructing possible minimal evolutionary histories. This problem was fi rst considered by Hein in [2], where unrooted trees were used. The main result of the work in [5] is that if the minimum number of recombination events is to be determined correctly for any data, then the right kind of trees and the right kind of distance between trees must be used. More exactly, the kind of trees which should be used are leaf-labelled rooted binary trees called ordered trees, in which internal vertices are totally ordered. Moreover, the induced SPR-metric on the space of ordered trees correctly quantifi es the number of recombination events, and therefore it is of interest to study SPR operations on ordered trees.

The focus of this paper is to study some properties of SPR operations on ordered trees. This paper is a sequel to our earlier paper [4], which considered similar questions for SPR operations on plain leaf-labelled rooted binary trees where internal vertices are only partially ordered. In comparison to the case of plain rooted trees, SPR operations on ordered trees are more diffi cult to study, and this fact is clearly reflected in our work. As in [4], we investigate the unit-neighbourhood $U(T)$, defi ned as the set of trees one SPR operation away from a given tree $T$. In relation to recombination, the unitneighbourhood size $|U(T)|$ gives the number of trees one recombination event away from $T$. To obtain an effi cient method of determining $|U(T)|$ for arbitrary tree topology, we construct a recursion relation, from which we are able to derive simple closed-form formulae for $|U(T)|$ for some special topology types. In [4], it was shown that for plain rooted trees the unit-neighbourhood size grows quadratically with respect to the number $n$ of leaves. In this paper, we show that $|U(T)|$ grows like $O\left(n^{3}\right)$ for ordered trees. We fi nd a sharp upper bound on $|U(T)|$, and using this result we construct bounds on the diameter of the space of ordered trees. In addition, we conjecture a formula for a sharp lower bound on $|U(T)|$.

This paper is organised as follows. In Section 2, we defi ne some notations and terminologies to be used throughout the paper. A more precise defi nition of SPR operations is provided there as well. The aforementioned recursion relation for $|U(T)|$ is constructed in Section 3, whereas special topology types with closed-form formulae for $|U(T)|$ are discussed in Section 4. In Section 5, we consider sharp bounds on $|U(T)|$. We conclude in Section 6 with a brief look into the diameter of the space of ordered trees. 
Note. We have written a computer program to check all our results for $n \leq 8$.

\section{Preliminaries}

In this section, we describe the kind of trees to be considered in this paper and introduce the notion of SPR operations on such trees. Some defi nitions are taken verbatim from [4].

\subsection{Trees}

In this paper, we consider leaf-labelled rooted binary trees whose branch lengths are not specifi ed. The discrete space of leaf-labelled rooted binary trees with $n$ leaves is denoted by $\mathscr{T}_{n}^{\mathrm{r}}$. To be distinguished from ordered trees, which we presently defi ne, a tree in $\mathscr{T}_{n}^{\mathrm{r}}$ is sometimes called a plain rooted tree. For $n \geq 2$, a tree in $\mathscr{T}_{n}^{\mathrm{r}}$ has $n$ labelled degree- 1 vertices called leaves; $n-2$ unlabelled degree- 3 vertices; and a distinguished vertex of degree 2 called the root. A 1-leaved tree consists of a single labelled degree- 0 vertex which serves as both the root and the leaf. The leaves of an $n$-leaved tree are bijectively labelled by a fi nite set $\mathcal{L}$ of $n$ elements. In the remainder of this paper, when we say a tree without any qualifi cation, we shall mean a leaf-labelled rooted binary tree.

An $n$-leaved rooted binary tree contains $2 n-2$ edges. A pendant edge is an edge incident with a leaf, and a cherry is a 2-leaved subtree. For any (sub)tree $s$, we denote by $\ell(s)$ the number of leaves in $s$. It was shown by Schröder [3] that the number of inequivalent leaf-labelled rooted binary trees with $n$ leaves is

$$
R(n):=\left|\mathscr{T}_{n}^{\mathrm{r}}\right|=(2 n-3) ! !=(2 n-3) \times(2 n-5) \times \cdots \times 3 \times 1=\frac{(2 n-2) !}{2^{n-1}(n-1) !} .
$$

In a rooted tree $T \in \mathscr{T}_{n}^{\mathrm{r}}$, time flows vertically from the root to the leaves, and we use $t(v)$ to denote the time associated to a vertex $v$. We say that a vertex $v \in T$ is a descendant of a vertex $u \in T$ if there exists a path from $u$ to $v$ which goes strictly forward in time; $u$ is called an ancestor of $v$. The set $\left\{v_{1}, v_{2}, \ldots, v_{n-2}\right\}$ of degree-3 vertices in $T \in \mathscr{T}_{n}^{\mathrm{r}}$ is a partially ordered set whose binary relation denoted $\preceq$ is given by ancestral relation; we say that $v_{i} \prec v_{j}$ if $v_{i}$ is an ancestor of $v_{j}$. Two degree-3 vertices $v_{i}$ and $v_{j}$ in $T \in \mathscr{T}_{n}^{\mathrm{r}}$ are incomparable if $v_{i}$ is not in the path to the root from $v_{j}$ and vice versa. An ordered tree is a leaf-labelled rooted binary tree whose corresponding set $\left\{v_{1}, v_{2}, \ldots, v_{n-2}\right\}$ of degree-3 vertices is a totally ordered set under the binary relation $\preceq_{a}$ defi ned by age ordering; we say that $u \prec_{a} v$ if and only if $t(u)<t(v)$. In an ordered tree, $t\left(v_{i}\right) \neq t\left(v_{j}\right)$ if $i \neq j$. Note that $v_{i} \prec_{a} v_{j}$ if $v_{j}$ is a descendant of $v_{i}$. If there exists no ancestral relation between $v_{i}$ and $v_{j}$, then either $v_{i} \prec_{a} v_{j}$ or $v_{j} \prec_{a} v_{i}$ is allowed. If $t(u)<t(v)$, or equivalently $u \prec_{a} v$, we say that $v$ is younger than $u$. All degree- 3 vertices in a tree are younger than the root. Two trees equivalent as plain rooted trees are distinct as ordered trees if the age ordering of their degree-3 vertices are different. For example, the two trees shown in Figure 1(a) are inequivalent ordered trees which are equivalent as plain rooted trees.

The parent $p(v)$ of a vertex $v$ is an ancestor of $v$ which is adjacent to $v$. Let $I(v)$ be the number of degree-3 vertices whose associated times lie between $t(v)$ and $t(p(v))$, i.e. $I(v)$ counts the number of intermediate degree-3 vertices between $t(v)$ and $t(p(v))$. For example, $I(u)=0$ and $I\left(u^{\prime}\right)=1$, where $u$ and $u^{\prime}$ are as indicated in Figure 1(a). Let 


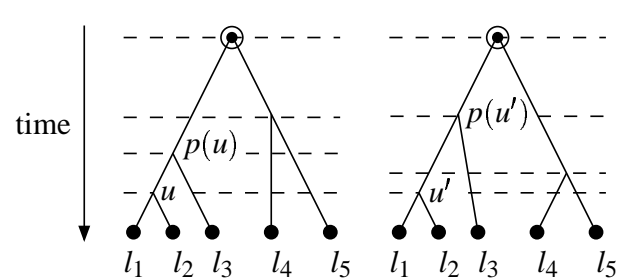

(a)

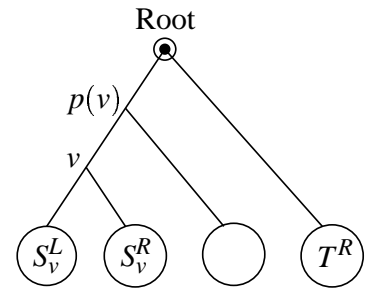

(b)

Figure 1: (a) Inequivalent ordered trees which are equivalent as plain rooted trees. (b) Illustration of some notations defined in the text.

$v$ be a vertex in $T$ of degree 2 or higher. Then, we use $S_{v}$ to denote the subtree whose root is $v$. Furthermore, we let $S_{v}^{L}$ and $S_{v}^{R}$ denote the two subtrees of $S_{v}$ whose roots are adjacent to $v$. By convention, $S_{v}^{L}$ (resp. $S_{v}^{R}$ ) is drawn on the left (resp. right). If $v$ is the root of $T$, then we defi ne $T^{L}:=S_{v}^{L}$ and $T^{R}:=S_{v}^{R}$. See Figure 1(b) for a schematic depiction of these defi nitions.

The space of ordered trees with $n$ leaves is denoted by $\mathscr{T}_{n}^{\circ}$, and the number of inequivalent ordered trees with $n$ leaves, for $n \geq 2$, is

$$
D(n):=\left|\mathscr{T}_{n}^{\mathrm{o}}\right|=\prod_{k=2}^{n}\left(\begin{array}{l}
k \\
2
\end{array}\right)=\frac{n !(n-1) !}{2^{n-1}} .
$$

This formula can be proved using induction on the number of leaves as follows. There exists a unique 2-leaved ordered tree and $D(2)=1$. Given $n$ labelled leaves, consider going backwards in time until exactly two leaves fi nd a common ancestor. There are $\left(\begin{array}{l}n \\ 2\end{array}\right)$ inequivalent such confi gurations. The number of inequivalent confi gurations further back in time is just the number of ordered trees with $n-1$ leaves. In summary, $D(n)=$ $\left(\begin{array}{l}n \\ 2\end{array}\right) D(n-1)=\left(\begin{array}{l}n \\ 2\end{array}\right) \prod_{k=2}^{n-1}\left(\begin{array}{l}k \\ 2\end{array}\right)=\prod_{k=2}^{n}\left(\begin{array}{c}k \\ 2\end{array}\right)$.

The number $\Omega(T)$ of ordered trees corresponding to a plain rooted tree $T$ depends on the topology of $T$. More exactly, the correspondence goes as follows. Let $d_{L}(v)$ (resp. $\left.d_{R}(v)\right)$ denote the number of degree-3 vertices which are left (resp. right) descendants of $v$. (Here, "left" and "right" refer to whether a descendant vertex is contained in $S_{v}^{L}$ or in $S_{v}^{R}$. In the tree shown on the left hand side of Figure 1(a), for example, $d_{L}($ root $)=2$ and $d_{R}($ root $)=1$, whereas $d_{L}(p(u))=1$ and $d_{R}(p(u))=0$.) Then, it is not diffi cult to show that

$$
\Omega(T)=\prod_{v, \text { vertices in } T \text { of degree } 2 \text { or higher }} \Delta(v),
$$

where $\Delta(v):=\left(d_{L}(v)+d_{R}(v)\right) ! /\left[d_{L}(v) ! d_{R}(v) !\right]$.

\subsection{SPR Operations on Plain Rooted Trees}

The precise defi nition of an SPR operation depends on the type of the tree on which the operation is being performed. In general, the more constraints a tree has, the more restrictive an SPR operation has to be. We begin our discussion with plain leaf-labelled rooted trees. There are three kinds of SPR operations that can be performed on leaflabelled rooted trees. Illustration of these operations is shown in Figure 2. In what follows, let $T$ (resp. $T^{\prime}$ ) denote a tree before (resp. after) an SPR operation. The notation 


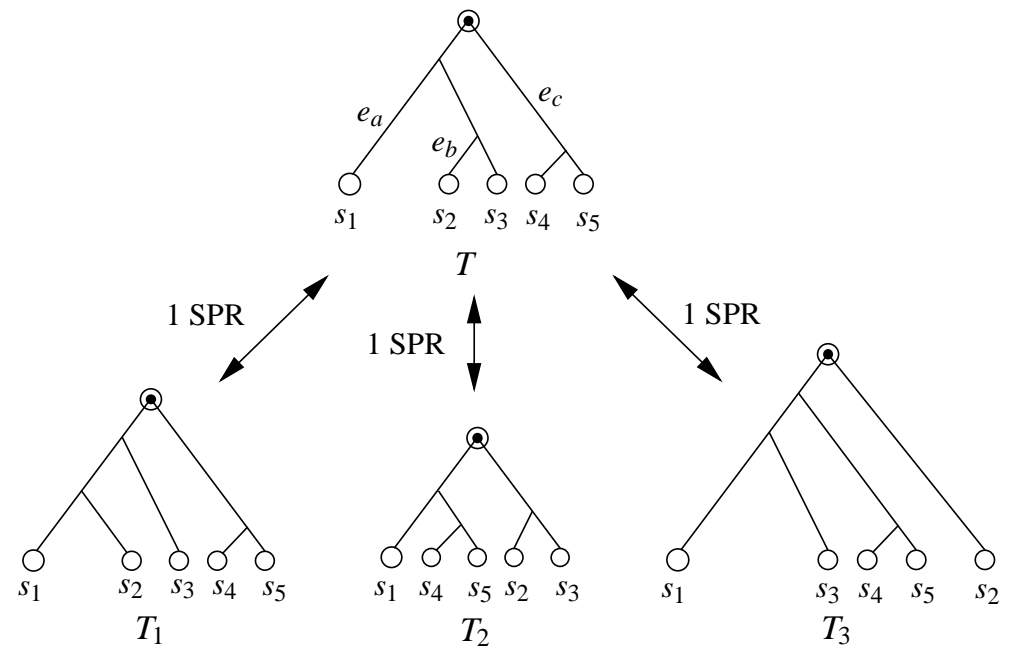

Figure 2: Illustration of SPR operations on plain rooted trees.

$T \backslash s$ denotes the part of $T$ obtained from removing a subtree $s$ and the edge incident with the root of $s$ but not contained in $s$. In words the three SPR operations are as follows.

(1) An edge $e$ is cut to prune a subtree $s$, not $T^{L}$ or $T^{R}$, and $s$ is regrafted onto a preexisting edge in the remaining part $T \backslash s$ of $T$, thus creating a new degree-3 vertex. The vertex in $T \backslash s$ where $e$ used to be incident gets removed. The root of $T$ remains the root of $T^{\prime}$. (In Figure 2, $T \rightarrow T_{1}$ is an example of this kind. The edge $e_{b}$ is cut and then regrafted onto the edge $e_{a}$.)

(2) Let $e_{L}$ and $e_{R}$, respectively, be the edges which join $T^{L}$ and $T^{R}$ to the root of $T$. The notations $L$ and $R$ can be interchanged in the following description: The edge $e_{L}$ is cut to prune $T^{L}$, and $T^{L}$ is regrafted onto a pre-existing edge in $T^{R}$. The edge $e_{R}$ gets removed and the vertex which used to be joined to the root of $T$ via $e_{R}$ becomes the root of $T^{\prime}$. (In Figure 2, $T \rightarrow T_{2}$ is an example of this kind. The edge $e_{c}$ can be cut and regrafted onto $e_{a}$. The root of $T^{L}$ containing $s_{1}, s_{2}$ and $s_{3}$ before the SPR operation then becomes the root of $T_{2}$.)

(3) Let $r$ denote the root of $T$. An edge $e$ is cut to prune a subtree $s$, not $T^{L}$ or $T^{R}$. A new root $r^{\prime}$ is created and an edge is formed from $r^{\prime}$ to $r$. Lastly, $s$ is joined to $r^{\prime}$. (In Figure 2, $T \rightarrow T_{3}$ is an example of this kind. The edge $e_{b}$ is cut and the pruned subtree $s_{2}$ gets joined to the new root. )

\subsection{SPR Operations on Ordered Trees}

For ordered trees, we impose an additional restriction on the defi nition of SPR operations. Consider a subtree $s$ of an ordered tree $T \in \mathscr{T}_{n}^{\circ}$. Let $u$ be the parent of the root of $s$. An operation which prunes and regrafts $s$ to transform $T \in \mathscr{T}_{n}^{\mathrm{o}}$ into $T^{\prime} \in \mathscr{T}_{n}^{\mathrm{o}}$ is defi ned to satisfy the following additional property: For any two vertices $v_{i}, v_{j} \in T$ neither being $u$, if $v_{i} \prec_{a} v_{j}$ before the SPR operation, then $v_{i} \prec_{a} v_{j}$ after the SPR operation, and vice versa. 

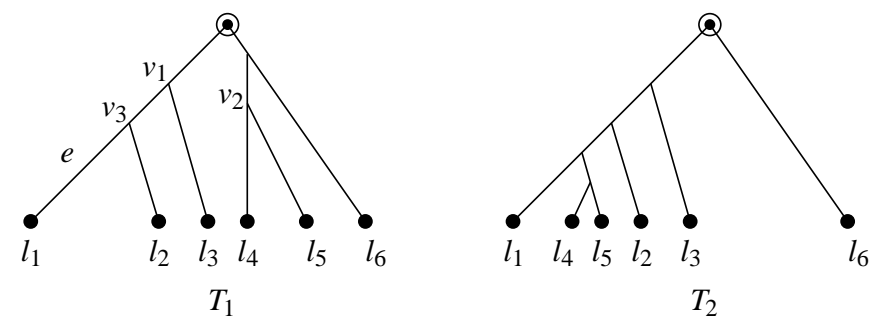

Figure 3: An example of ordered trees which are more than one SPR operation apart.

The two ordered trees $T_{1}$ and $T_{2}$ shown in Figure 3 are more than one SPR operation away from each other. If $T_{1}$ and $T_{2}$ were plain rooted trees, then the subtree containing $l_{4}$ and $l_{5}$ could be pruned and regrafted onto the edge $e$ to transform $T_{1}$ into $T_{2}$. Such an operation is forbidden for ordered trees, however, because the ordering of the degree- 3 vertices $v_{2}$ and $v_{3}$ would change by the operation. As a result, at least 2 SPR operations are required to transform $T_{1}$ into $T_{2}$, and vice versa.

\section{The Unit-Neighbourhood of an Ordered Tree}

Let $d_{S P R}\left(T, T^{\prime}\right)$ denote the minimum number of SPR operations required to transform $T$ into $T^{\prime}$. The unit-neighbourhood of an $n$-leaved ordered tree $T$ is defi ned as

$$
U(T)=\left\{T^{\prime} \in \mathscr{T}_{n}^{\mathrm{o}} \mid d_{S P R}\left(T, T^{\prime}\right)=1\right\}
$$

In this section, we discuss how the size $|U(T)|$ can be computed in a systematic way.

\subsection{Moving Below the Youngest Degree-3 Vertex}

Consider an $n$-leaved ordered tree $T$. The part of the tree below the youngest degree3 vertex is illustrated in Figure 4. We are interested in knowing how many inequivalent ordered trees can be obtained by pruning a leaf labelled $l_{i} \in \mathcal{L} \backslash\left\{l_{n-1}, l_{n}\right\}$ and regrafting it onto a pendant edge below level $t$ but not on the cherry containing $l_{n-1}$ and $l_{n}$. There are $(n-2)$ ways of choosing a leaf for pruning and $(n-3)$ ways of choosing a pendant edge for regrafting. But, not all $(n-2)(n-3)$ such SPR operations lead to distinct ordered trees, and the resulting number of inequivalent ordered trees depends on the topology of the original tree $T$. Let us examine how over-counting can arise.

Perhaps the best way to illustrate how the above-mentioned counting should work is through an explicit example. Consider the example shown in Figure 5, where, for ease

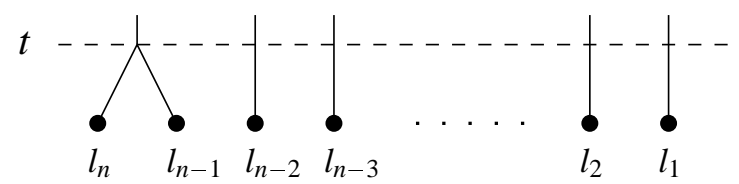

Figure 4: Schematic depiction of the bottom of an $n$-leaved ordered tree. The youngest degree- 3 vertex in the tree is the one to which $l_{n-1}$ and $l_{n}$ are joined to form a cherry. 


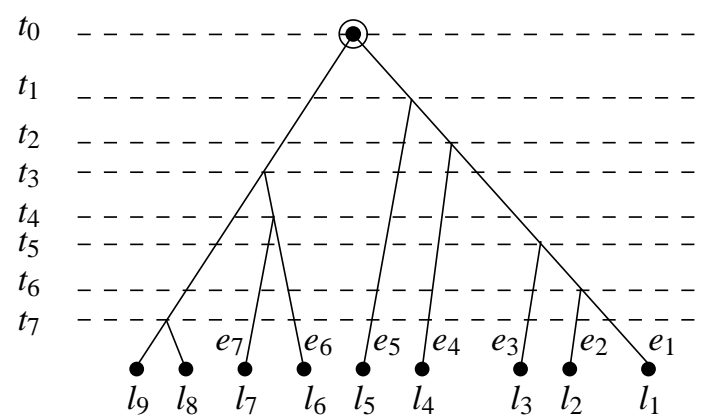

Figure 5: An example of a 9-leaved ordered tree where the problem of over-counting described in Section 3.1 can arise in several ways. For some pendant edges, the part below $t_{7}$ is labelled according to the leaf label.

of discussion, we have given labels to the parts below $t_{7}$ for some pendant edges. To avoid being long-winded, let $l_{i} \rightsquigarrow e_{j}$ denote pruning $l_{i}$ and regrafting it onto $e_{j}$ below level $t_{7}$. Then, note that $l_{6} \leadsto e_{7}$ leads to the same ordered tree as that obtained from $l_{7} \rightsquigarrow e_{6}$. Likewise, $l_{1} \rightsquigarrow e_{2}$ is equivalent to $l_{2} \rightsquigarrow e_{1}$. More generally, the number of overcounting due to this kind of symmetry is $c(T)-1$, where $c(T)$ is the number of cherries in $T$; here, -1 is for the cherry containing $l_{n}$ and $l_{n-1}$.

Over-counting can arise in another way as well. For example, $l_{4} \rightsquigarrow e_{5}$ is equivalent to $l_{5} \rightsquigarrow e_{4}$. Similarly, $l_{1} \rightsquigarrow e_{3}$ is equivalent to $l_{3} \rightsquigarrow e_{1}$ and $l_{2} \rightsquigarrow e_{3}$ is equivalent to $l_{3} \rightsquigarrow e_{2}$. It is important to note, however, that $l_{3} \rightsquigarrow e_{4}$ is not equivalent to $l_{4} \rightsquigarrow e_{3}$. The presence of intermediate degree- 3 vertices between $t_{2}$ and $t_{5}$ distinguishes the ordered tree obtained by $l_{3} \rightsquigarrow e_{4}$ from that obtained by $l_{4} \rightsquigarrow e_{3}$. More generally, the number of over-counting due to this kind of symmetry is equal to the number of leaves, other than $l_{n}$ and $l_{n-1}$, satisfying the following: Let $v$ denote the parent vertex of the leaf. Then, $(i)$ the parent $p(v)$ of $v$ is incident with a pendant edge, and (ii) there is no intermediate degree-3 vertex between $t(v)$ and $t(p(v))$. The number of such leaves is given by summing the following quantity over all degree- 3 vertices $v$ except for the youngest one, which is the root of the cherry containing $l_{n}$ and $l_{n-1}$ :

$$
w(v):=\delta_{I(v), 0} \delta_{\left.\ell\left(S_{p(v)}\right) S_{v}\right), 1}\left[\delta_{\ell\left(S_{v}^{L}\right), 1}+\delta_{\ell\left(S_{v}^{R}\right), 1}\right] .
$$

Here, $\delta_{a, b}$ is the Kronecker delta function and the remaining notations have been defi ned in Section 2.1. The fi rst delta function in (3.2) makes sure that there are no intermediate degree-3 vertices between $t(v)$ and $t(p(v))$; the second delta function makes sure that the parent vertex $p(v)$ is incident with a pendant edge; the last delta function counts the number of pendant edges incident with $v$.

It is straightforward to check that there are no other sources of over-counting. In summary, the number of inequivalent ordered trees obtained from the SPR operations being considered here is

$$
(n-2)(n-3)-\left[c(T)-1+\sum_{v \neq \text { youngest }} w(v)\right]=:(n-2)(n-3)-b(T),
$$




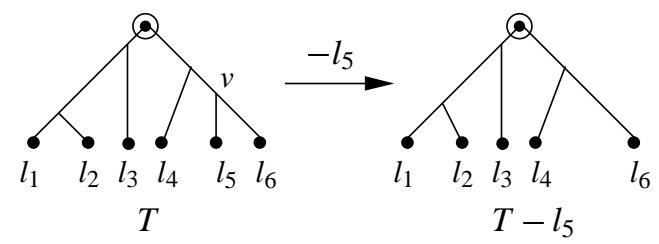

Figure 6: An example of pruning without regrafting for a 6-leaved ordered tree.

where the sum is over all degree-3 vertices except for the youngest one. Just to demonstrate how this works, let us return to the example shown in Figure 5. Let $v_{i}$ denote the vertex at $t_{i}$. Then, one obtains

$$
w\left(v_{1}\right)=0, w\left(v_{2}\right)=1, w\left(v_{3}\right)=0, w\left(v_{4}\right)=0, w\left(v_{5}\right)=0, w\left(v_{6}\right)=2 .
$$

Therefore, since $n=9$ and $C(T)=3$ in the present example, $(n-2)(n-3)-b(T)=$ $(9-2)(9-3)-(3-1+1+2)=37$, which, as one can check explicitly, is the correct answer.

\subsection{Pruning without Regrafting}

We here defi ne an operation which reduces the number of leaves in a tree by one. In an $n$-leaved ordered tree $T$, let $v$ denote a degree- 3 vertex with a leaf labelled $l_{k}$ adjacent to it. Then, $T-l_{k}$ is defi ned as an $(n-1)$-leaved tree obtained by removing $v, l_{k}$ and the edge joining them, and then connecting the two other edges which used to be incident with $v$ into a single edge. An example is shown in Figure 6.

\subsection{A Recursion for $|U(T)|$ : The Bottom-Up Approach}

Proposition 3.1. For $n \geq 4$ and $T \in \mathscr{T}_{n}^{\circ}$, the size of the unit-neighbourhood $U(T)$ satisfies the recursion relation

$$
|U(T)|=2\left(n^{2}-2 n-2\right)-(h-1)\left(1-\delta_{h, 0}\right)-b(T)+\left|U\left(T-l_{n}\right)\right|,
$$

where $l_{n}$ is a leaf with its parent vertex $p\left(l_{n}\right)$ being the youngest degree-3 vertex in $T$, $b(T)$ is defined as in (3.3), and $h:=I\left(p\left(l_{n}\right)\right)$, i.e. the number of intermediate degree-3 vertices between $t\left(p\left(l_{n}\right)\right)$ and $t\left(p\left(p\left(l_{n}\right)\right)\right)$.

Remark. Note that $|U(T)|=2$, for all $T \in \mathscr{T}_{3}^{\circ}$, serves as the boundary condition for the recursion.

Proof. There are 3 distinct cases we need to consider. These cases are illustrated in Figure 7. In each case, let $v$ be the youngest degree- 3 vertex in $T$; that is, let $v$ be the parent vertex of $l_{n}$. We first do a "coarse counting" of the number of additional moves which are made possible because of the presence of $v$, i.e. we want to count the number of moves which would be absent if the cherry containing $l_{n-1}$ and $l_{n}$ were instead a single-leaved subtree. Then, for each of the 3 cases illustrated in Figure 7, we shall analyse which moves included in the "coarse counting" lead to equivalent ordered trees, thus eliminating all over-counting.

The following "coarse counting" of moves is common to all three cases: 


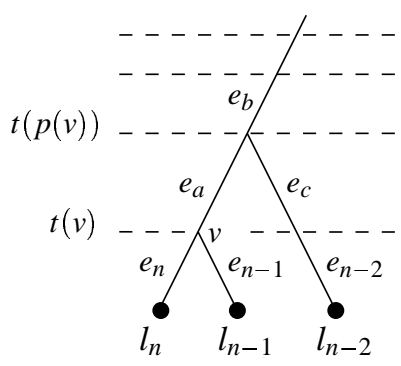

(a)

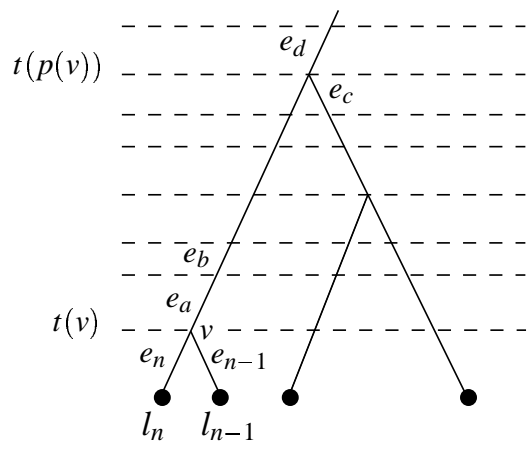

(b)

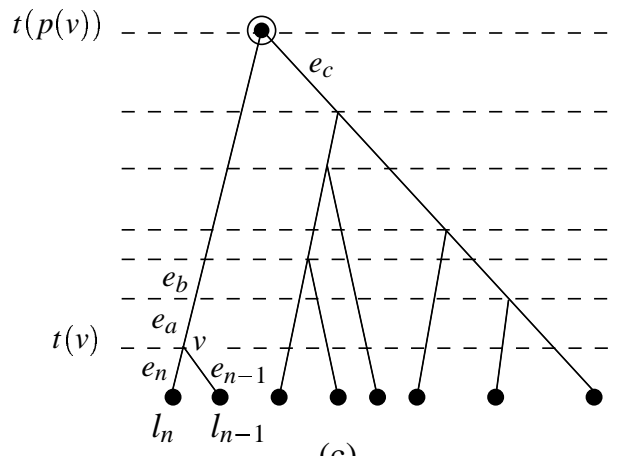

(c)

Figure 7: Illustration of the three possible cases in the proof of Proposition 3.1. (a) $h=0$. (b) $h \neq 0$ and $p(v)$ is not the root. (c) $h \neq 0$ and $p(v)$ is the root.

(a) $+2(n-2)$ from moving other pendant edges to $e_{n}$ or $e_{n-1}$.

(b) $+\left(n^{2}-n-2\right)$ from cutting $e_{n}$ or $e_{n-1}$ and then attaching it somewhere above $t(v)$. There are $\sum_{k=1}^{n-2}(k+1)=\left(n^{2}-n-2\right) / 2$ distinct regions above $t(v)$, depending on which edge and which time interval is chosen. Note that moving $e_{n}$ or $e_{n-1}$ to somewhere below $t(v)$ leads to a tree topology already included here.

(c) +2 from cutting $e_{n}$ or $e_{n-1}$ and then attaching it to the root.

(d) $+(n-2)(n-3)-b(T)$ inequivalent moves from pruning any of $l_{1}, \ldots, l_{n-2}$ and then regrafting it somewhere below $t(v)$ but not onto $e_{n}$ or $e_{n-1}$. These moves have been discussed in Section 3.1.

So far we have counted $2(n-2)+\left(n^{2}-n-2\right)+2+(n-2)(n-3)-b(T)=$ $2(n-1)^{2}-b(T)$ moves, not all of which are inequivalent. We now account for possible over-counting. In each case, the notation used conforms to the corresponding fi gure.

Case $1(h=0)$ :

In this case, since $v$ is the youngest degree-3 vertex, the descendant subtree of $p(v)$ not containing $v$ must contain exactly one leaf. Moreover, since $n \geq 4, p(v)$ cannot be the root. We refer to Figure 7(a) in the following discussion:

(1-1) -2 : Cutting $e_{n}$ or $e_{n-1}$ and then attaching it to $e_{a}$ does not change the topology. 
(1-2) -4 : Moving $e_{n}$ (resp. $e_{n-1}$ ) to $e_{c}$ is equivalent to pruning $l_{n-2}$ and regrafting it onto $e_{n}$ (resp. $e_{n-1}$ ). Also, moving $e_{n}$ (resp. $\left.e_{n-1}\right)$ to $e_{b}$ is equivalent to pruning $l_{n-2}$ and then regrafting it onto $e_{n-1}\left(\operatorname{resp} . e_{n}\right)$. These lead to double-counting in (a) and (b) of the above list.

Case $2(h \neq 0$ and $p(v)$ is not the root $)$ :

Shown in Figure 7(b) is a partial depiction of an ordered tree which falls into this case.

(2-1) -2 : Cutting $e_{n}$ or $e_{n-1}$ and then attaching it to $e_{a}$ does not change the topology.

(2-2) -2 : Cutting $e_{n}$ or $e_{n-1}$ and then attaching it to $e_{b}$ leads to a tree topology also included in part (d) of the above list.

(2-3) -2 : Moving $e_{n}$ (resp. $e_{n-1}$ ) to $e_{c}$ is equivalent to moving $e_{n-1}$ (resp. $e_{n}$ ) to $e_{d}$.

(2-4) $-(h-1)$ : Let $E$ be the edge joining $v$ with $p(v)$. Pruning $l_{n}$ and then regrafting it somewhere on $E$ is equivalent to doing the same thing to $l_{n-1}$. Other than $e_{a}$ and $e_{b}$, which we have already considered above, there are $h-1$ intervals in $E$.

Case 3 ( $h \neq 0$ and $p(v)$ is the root):

An example of this case is shown in Figure 7(c).

(3-1) -2 : Cutting $l_{n}$ or $l_{n-1}$ and then attaching it to $e_{a}$ does not change the topology.

(3-2) -2 : Cutting $e_{n}$ or $e_{n-1}$ and then attaching it to $e_{b}$ leads to a tree topology also included in part (d) of the above list.

(3-3) -2 : Moving $e_{n}$ (resp. $e_{n-1}$ ) to $e_{c}$ is equivalent to moving $e_{n-1}$ (resp. $e_{n}$ ) to the root. This leads to double-counting in (b) and (c) of the above list.

(3-4) $-(h-1)$ : Let $E$ be the edge joining $v$ with $p(v)$. Pruning $l_{n}$ and then regrafting it somewhere on $E$ is equivalent to doing the same thing to $l_{n-1}$. Other than $e_{a}$ and $e_{b}$, which we have already considered above, there are $h-1$ intervals in $E$.

In summary, over-counting in each case contributes $-\left[6+(h-1)\left(1-\delta_{h, 0}\right)\right]$, and thus the number of inequivalent ordered trees in $U(T)$ which arise due to the presence of $v$ is $2(n-1)^{2}-b(T)-\left[6+(h-1)\left(1-\delta_{h, 0}\right)\right]$, which can be written as $2\left(n^{2}-2 n-2\right)$ $\left.-(h-1)\left(1-\delta_{h, 0}\right)\right]-b(T)$. The remaining number of inequivalent ordered trees in $U(T)$ is given by $\left|U\left(T-l_{n}\right)\right|$. This completes our proof of the proposition.

\subsection{Solving for $|U(T)|$}

The recursion shown in (3.4) can be carried out sequentially until a 3-leaved ordered tree is reached. Many terms in such an expansion can be summed explicitly to obtain a simpler formula for $|U(T)|$. Before we proceed, we introduce an additional notation which will shortly prove convenient. Let $l$ be a leaf adjacent with the youngest vertex in an ordered tree $T$. Then, $P(T)$ is defi ned as the ordered tree $T-l$ obtained by pruning $l$ from $T$. In a similar vein, $\mathcal{P}^{k}(T)$ is recursively defi ned by pruning from $\mathcal{P}^{k-1}(T)$ a leaf adjacent to the youngest vertex in $P^{k-1}(T)$. The initial condition is $P^{0}(T)=T$.

Now, since $\left|U\left(T^{\prime}\right)\right|=2$ for all 3-leaved ordered trees $T^{\prime}$ and $2+\sum_{k=4}^{n} 2\left(k^{2}-2 k-2\right)$ $=\frac{1}{3}(n+3)(n-2)(2 n-5)$, the unit-neighbourhood size of $T$ with degree-3 vertices $\left\{v_{1}, \ldots, v_{n-2}\right\}$ can be written as

$$
\begin{aligned}
|U(T)|= & \frac{1}{3}(n+3)(n-2)(2 n-5)-\sum_{i=1}^{n-2}\left(I\left(v_{i}\right)-1\right)\left(1-\delta_{I\left(v_{i}\right), 0}\right) \\
& -\sum_{k=0}^{n-4}\left[c\left(\mathcal{P}^{k}(T)\right)-1+w\left(\mathcal{P}^{k}(T)\right)\right] .
\end{aligned}
$$




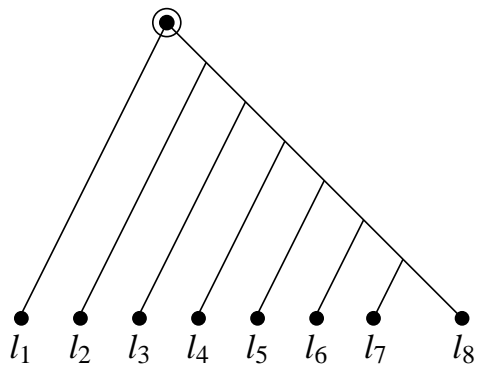

(a)

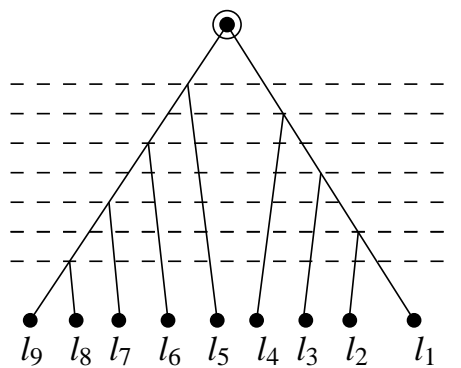

(b)

Figure 8: Examples of special topology types with closed-form formulae for $|U(T)|$. (a) An 8leaved caterpillar tree. (b) A 9-leaved alternating-comb tree.

Here, $c\left(\mathcal{P}^{k}(T)\right)$ is the number of cherries in $\mathcal{P}^{k}(T)$ and $w\left(\mathcal{P}^{k}(T)\right)$ denotes

$$
\sum_{v, \text { not the youngest in } P^{k}(T)} w(v),
$$

where $w(v)$ is defi ned as in (3.2). We observe that (3.5) is considerably more complicated than the corresponding formula for plain rooted trees (c.f. Proposition 3.2. of [4]). Also, because the negative terms in (3.5) are at most of $O\left(n^{2}\right)$, the unit-neighbourhood size $|U(T)|$ for an ordered tree $T$ is of $O\left(n^{3}\right)$. This is in contrast to the case of plain rooted trees, where the unit-neighbourhood size grows quadratically with respect to $n$.

\section{Some Special Cases with Closed-Form Formulae}

The formula for $|U(T)|$ shown in (3.5) is not in closed-form, but it may take on a simple form if we focus on a specifi c topology type. In this section, we consider three special topology types with closed-form formulae for $|U(T)|$. Results from this section will be used in the next section.

\subsection{Caterpillar Trees}

A caterpillar tree is a tree of the type illustrated in Figure 8(a). We have the following result for the size of the unit-neighbourhood:

Proposition 4.1. Let $n \geq 3$. For an $n$-leaved caterpillar tree $T$,

$$
|U(T)|=\frac{1}{6}\left(4 n^{3}-9 n^{2}-19 n+42\right) .
$$

Proof. Let us analyse the unevaluated sums appearing in (3.5). Note that $I\left(v_{i}\right)=0$, and therefore $\left(1-\delta_{I\left(v_{i}\right), 0}\right)=0$, for all degree-3 vertices $v_{i}$ in a caterpillar tree. Moreover, since a caterpillar tree contains exactly one cherry and since if $T$ is a caterpillar tree, then so is $\mathcal{P}^{k}(T)$, we conclude that $c\left(\mathcal{P}^{k}(T)\right)=1$ for all $0 \leq k \leq n-4$. Finally, since $w\left(T^{\prime}\right)=j-3$ for a $j$-leaved caterpillar tree, (3.5) is equal to $\frac{1}{3}(n+3)(n-2)(2 n-5)-$ $\sum_{j=4}^{n}(j-3)=\frac{1}{6}\left(4 n^{3}-9 n^{2}-19 n+42\right)$, which is our desired result. 
In [4], it was shown that, for $\tau$ an $n$-leaved caterpillar tree regarded as a plain rooted tree, the unit-neighbourhood size $|U(\tau)|$ is given by $3 n^{2}-13 n+14$.

\subsection{Alternating-Comb Trees}

We here defi ne a new topology type which contains two caterpillar trees as subtrees. An example of this new topology type is shown in Figure 8(b). More exactly, an alternatingcomb tree $T$ is defi ned as an ordered tree such that $T^{L}$ (resp. $T^{R}$ ) is a caterpillar tree with $\left\lceil\frac{n}{2}\right\rceil$ (resp. $\left\lfloor\frac{n}{2}\right\rfloor$ ) leaves, and the age-ordered sequence of degree- 3 vertices in $T$ alternates between $T^{L}$ and $T^{R}$. Here, $\lceil\cdot\rceil$ is the ceiling function, whereas $\lfloor\cdot\rfloor$ is the floor function. The following proposition shows that alternating-comb trees also admit a very simple closed-form formula for the unit-neighbourhood size.

Proposition 4.2. Let $n \geq 3$. For an $n$-leaved alternating-comb tree $T$,

$$
|U(T)|=\frac{1}{3}\left(2 n^{3}-3 n^{2}-20 n+39\right) .
$$

Proof. Let $4 \leq m \leq n$ and let $\left\{v_{1}, v_{2}, \ldots, v_{m-2}\right\}$ label the set of all degree- 3 vertices in an $m$-leaved alternating-comb tree $T^{\prime}$ so that $t\left(v_{1}\right)<t\left(v_{2}\right)<\cdots<t\left(v_{m-2}\right)$. Then, $I\left(v_{1}\right)=0$ and $I\left(v_{i}\right)=1$ for all $2 \leq i \leq m-2$, and therefore $\left(I\left(v_{i}\right)-1\right)\left(1-\delta_{I\left(v_{i}\right), 0}\right)=0$ for all $1 \leq i \leq m-2$. Furthermore, from the defi nition of $w(v)$ in (3.2), we conclude that $w\left(v_{1}\right)=0$, since $\ell\left(S_{p\left(v_{1}\right)} \backslash S_{v_{1}}\right)>1$ for an $m$-leaved alternating-comb tree where $m \geq 4$. Also, for all $2 \leq i \leq m-2$, we have $w\left(v_{i}\right)=0$ because $\delta_{I\left(v_{i}\right), 0}=0$. Lastly, we note that $c\left(T^{\prime}\right)=2$.

Now, since $\mathcal{P}^{k}(T)$, for all $0 \leq k \leq n-4$, also is an alternating-comb tree if $T$ is an alternating-comb tree, the above discussion leads to the following non-vanishing contributions to (3.5):

$$
\begin{aligned}
|U(T)| & =\frac{1}{3}(n+3)(n-2)(2 n-5)-\left[\sum_{k=0}^{n-4} c\left(\mathcal{P}^{k}(T)\right)-1\right] \\
& =\frac{1}{3}(n+3)(n-2)(2 n-5)-(n-3),
\end{aligned}
$$

which is equal to the (4.7).

\subsection{Cherry-Descending Trees}

Consider the $n$-leaved ordered tree shown in Figure 9. It contains $\left\lfloor\frac{n-1}{2}\right\rfloor$ cherries, of which $\left\lfloor\frac{n-1}{2}\right\rfloor-1$ are ordered in sequentially decreasing order as shown in the fi gure. Note that the value of the expression $n+1-2\left\lfloor\frac{n-1}{2}\right\rfloor$ shown in the fi gure is 2 if $n$ is odd or 3 if $n$ is even. For $n=3$ and $n=4$, cherry-descending trees and caterpillar trees are the same.

Proposition 4.3. Let $n \geq 3$. For an $n$-leaved cherry-descending tree $T$,

$$
|U(T)|=\frac{1}{6}\left\{4 n^{3}-9 n^{2}-13 n+42-3(2 n+3)\left\lfloor\frac{n-1}{2}\right\rfloor+9\left(\left\lfloor\frac{n-1}{2}\right\rfloor\right)^{2}\right\} .
$$




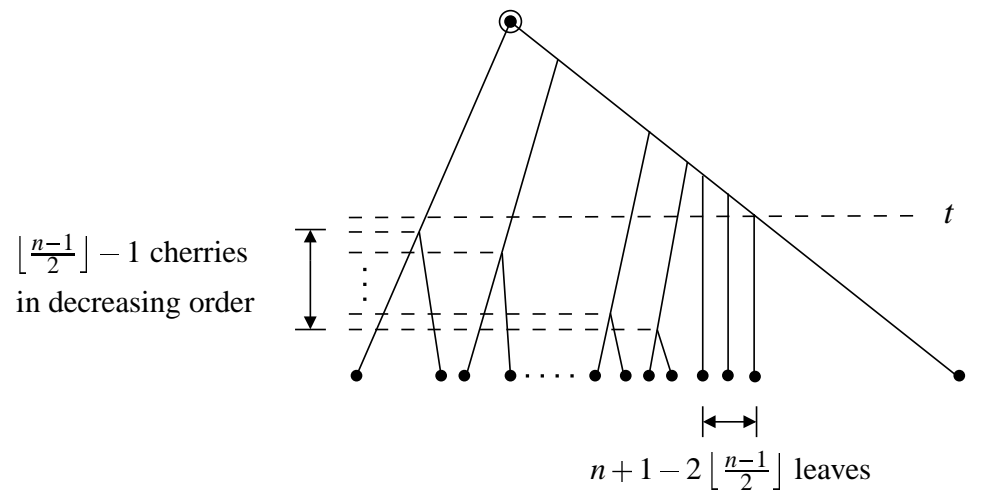

Figure 9: An $n$-leaved cherry-descending tree. There are $\left\lfloor\frac{n-1}{2}\right\rfloor$ cherries in this tree.

Proof. We apply the main recursion (3.4) in the bottom-up fashion until what is left is a caterpillar tree $T^{\prime}$ with $n-\left(\left\lfloor\frac{n-1}{2}\right\rfloor-1\right)$ leaves. From Proposition 4.1, we know $\left|U\left(T^{\prime}\right)\right|$. We wish to examine what the other terms in the recursion contribute to $|U(T)|$. In the following discussion, we keep in mind that the number of times that the recursion must be applied before $T^{\prime}$ is reached is $\left(\left\lfloor\frac{n-1}{2}\right\rfloor-1\right)$. Adding up the terms like the fi rst one on the right hand side of (3.4) gives

$$
\sum_{i=n-\left(\left\lfloor\frac{n-1}{2}\right\rfloor-1\right)+1}^{n} 2\left(i^{2}-2 i-2\right) .
$$

Furthermore, since $I(v)=n-\left\lfloor\frac{n-1}{2}\right\rfloor-1$, for all degree-3 vertices $v$ below $t$, the sum of the terms like $-(h-1)\left(1-\delta_{h, 0}\right)$ gives

$$
-\left(n-\left\lfloor\frac{n-1}{2}\right\rfloor-2\right)\left(\left\lfloor\frac{n-1}{2}\right\rfloor-1\right) .
$$

Lastly, one can show that $w\left(\mathcal{P}^{k}(T)\right)=n-2\left\lfloor\frac{n-1}{2}\right\rfloor+1+k$ and that $c\left(\mathcal{P}^{k}(T)\right)-1=$ $\left\lfloor\frac{n-1}{2}\right\rfloor-1-k$, thus yielding

$$
-\sum_{k=0}^{\left\lfloor\frac{n-1}{2}\right\rfloor-2} b\left(\mathcal{P}^{k}(T)\right)=-\left(n-\left\lfloor\frac{n-1}{2}\right\rfloor\right)\left(\left\lfloor\frac{n-1}{2}\right\rfloor-1\right) \text {. }
$$

Summing $\left|U\left(T^{\prime}\right)\right|$, (4.9), (4.10) and (4.11) gives (4.8).

\section{Sharp Bounds on $|U(T)|$}

In this section, we study sharp lower and upper bounds on $|U(T)|$. We defi ne

$$
\delta_{\min }(n)=\min _{T \in \mathscr{T}_{n}}|U(T)| \quad \text { and } \quad \delta_{\max }(n)=\max _{T \in \mathscr{T}_{n}}|U(T)|,
$$




\begin{tabular}{||r||r|r|r||r|r|r||}
\hline \multicolumn{1}{||c||}{} & \multicolumn{3}{c||}{ Plain Rooted Trees } & \multicolumn{3}{c||}{ Ordered Trees } \\
\hline \hline$n$ & $R(n)$ & $\delta_{\min }(n)$ & $\delta_{\max }(n)$ & $D(n)$ & $\delta_{\min }(n)$ & $\delta_{\max }(n)$ \\
\hline 3 & 3 & 2 & 2 & 3 & 2 & 2 \\
\hline 4 & 15 & 10 & 12 & 18 & 13 & 13 \\
\hline 5 & 105 & 24 & 28 & 180 & 35 & 38 \\
\hline 6 & 945 & 44 & 52 & 2,700 & 75 & 81 \\
\hline 7 & 10,395 & 70 & 84 & 56,700 & 135 & 146 \\
\hline 8 & 135,135 & 102 & 124 & $1,587,600$ & 220 & 237 \\
\hline
\end{tabular}

Table 1. The minimum and the maximum values of $|U(T)|$ for plain rooted trees and for ordered trees. These have been determined via computer-aided exhaustive search.

where $\mathscr{T}_{n}$ is either $\mathscr{T}_{n}^{\mathrm{o}}$ or $\mathscr{T}_{n}^{\mathrm{r}}$, depending on whether plain rooted trees or ordered trees are being considered. We have written a computer program which computes the unitneighbourhood size $|U(T)|$ through exhaustive comparison of trees, and therefore, for small number $n$ of leaves, the minimum and the maximum values of $|U(T)|$ can be determined via explicit computation. Table 1 shows the result of such computation for $n \leq 8$.

In [4], closed-form formulae for $\delta_{\min }(n)$ and $\delta_{\max }(n)$ were derived for plain rooted trees, and they agree with our exhaustive search results summarised in the fir rst part of Table 1. The goal of this section is to construct closed-form formulae for the numbers shown in the second part of Table 1 pertaining to ordered trees. ${ }^{1}$

\subsection{The Maximum Unit-Neighbourhood Size}

We fi rst establish the following proposition regarding alternating-comb trees defi ned in Section 4.2:

Proposition 5.1. In the case of ordered trees with $n$ leaves, where $n \geq 3$, alternatingcomb trees have the maximum unit-neighbourhood size.

Proof. We shall prove this statement by induction on $n$. For $n=3$ and $n=4$, all ordered trees have $|U(T)|=2$ and $|U(T)|=13$, respectively. For $n=5$, one can explicitly show that a 5-leaved alternating-comb tree $T$ has $|U(T)|=38$, which is the maximum value. Suppose that the statement in the proposition is true for all $3 \leq n \leq k-1$, where $k \geq 6$. We now use (3.4) to compute $|U(T)|$, where $T$ is a $k$-leaved alternating-comb tree. Let $l_{k}$ be a leaf adjacent with the youngest vertex in $T$. Then, we note that $T-l_{k}$ also is an alternating-comb tree if $T$ is an alternating-comb tree. It therefore follows from the induction hypothesis that $\left|U\left(T-l_{k}\right)\right|=\delta_{\max }(k-1)$. Furthermore, because $I\left(p\left(l_{k}\right)\right)=1$ and $b(T)=1$ if $T$ is a $k$-leaved alternating-comb tree, the remaining terms in (3.4) contribute $2\left(k^{2}-2 k-2\right)-1$. In summary, $|U(T)|=\delta_{\max }(k-1)+2\left(k^{2}-2 k-2\right)-1$.

Now, suppose that $T^{\prime}$ is an arbitrary $k$-leaved ordered tree and that $l_{k}$ be a leaf adjacent with the youngest vertex in $T^{\prime}$. Then, it follows from (3.4) that

$$
\left|U\left(T^{\prime}\right)\right|=\left|U\left(T^{\prime}-l_{k}\right)\right|+2\left(k^{2}-2 k-2\right)-\left(I\left(p\left(l_{k}\right)\right)-1\right)\left(1-\delta_{I\left(p\left(l_{k}\right)\right), 0}\right)-b\left(T^{\prime}\right) .
$$

\footnotetext{
1 Incidentally, we take this opportunity to report an error in Table 1 of [5], which shows incorrect values of $\delta_{\min }(n)$ and $\delta_{\max }(n)$ for ordered trees. In that work, ordered trees in $|U(T)|$ which are equivalent to $T$ as plain rooted trees were accidentally omitted in the exhaustive search.
} 

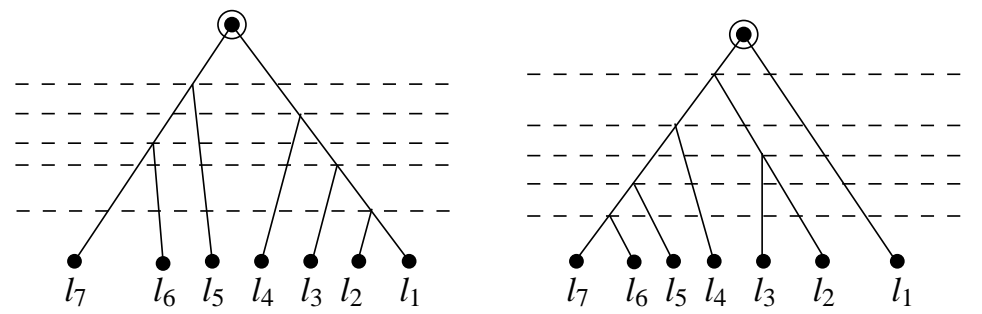

Figure 10: 7-leaved ordered trees which are not alternating-comb trees but still with the maximum unit-neighbourhood size.

Clearly, $\left(I\left(p\left(l_{k}\right)\right)-1\right)\left(1-\delta_{I\left(p\left(l_{k}\right)\right), 0}\right)$ is non-negative. Furthermore, every tree contains at least one cherry, so $c\left(T^{\prime}\right) \geq 1$. Suppose that $c\left(T^{\prime}\right)=1$. Then, $T^{\prime}$ must be a caterpillar tree, so $b\left(T^{\prime}\right)=w\left(T^{\prime}\right)=k-3$, which is greater than 1 since $k \geq 6$. Suppose that $c\left(T^{\prime}\right)>$ 1 . Then, clearly $b\left(T^{\prime}\right)=c\left(T^{\prime}\right)-1+w\left(T^{\prime}\right) \geq 1$, since $w\left(T^{\prime}\right)$ is non-negative. Thus, for all $k$-leaved ordered trees $T^{\prime}$, we must have $b\left(T^{\prime}\right) \geq 1$. Combining this result with the fact that $\left|U\left(T^{\prime}-l_{k}\right)\right| \leq \delta_{\max }(k-1)$, we conclude that $\left|U\left(T^{\prime}\right)\right| \leq|U(T)|$. In other words, $|U(T)|=\delta_{\max }(k)$. This completes our induction.

The following sharp upper bound on $|U(T)|$ now follows straightforwardly from Proposition 4.2 and Proposition 5.1:

Corollary 5.2. For ordered trees with $n$-leaves, where $n \geq 3$,

$$
\delta_{\max }(n)=\frac{1}{3}\left(2 n^{3}-3 n^{2}-20 n+39\right) .
$$

As it should be, the formula given in (5.12) is consistent with the numerical values obtained from our computer-aided exhaustive search (c.f. Table 1). Also, we point out that alternating-comb trees are not the only type of trees with the maximum unitneighbourhood size. For example, the 7-leaved ordered trees shown in Figure 10 also have $\delta_{\max }(7)=146$ as their unit-neighbourhood size.

\subsection{The Minimum Unit-Neighbourhood Size}

For plain rooted trees, it was shown in [4] that caterpillar trees have the minimum unit-neighbourhood size. For ordered trees, however, that no longer holds true. For example, for $n \geq 5$, the formula for cherry-descending trees (c.f. (4.8)) always leads to a smaller value than that for caterpillar trees (c.f. (4.6)). In fact, the formula shown in (4.8) agrees with our exhaustively-determined numerical values of $\delta_{\min }(n)$ for ordered trees shown in Table 1. Moreover, for $n \leq 8$, we have explicitly checked that an ordered tree has the minimum unit-neighbourhood size if and only if it is a cherry-descending tree. This is in contrast with the maximum size case, where several different topology types can have the maximum unit-neighbourhood size.

It does not seem straightforward to show that cherry-descending trees have the minimum unit-neighbourhood size for all $n \geq 3$. Hence, based on the successful match, for $n \leq 8$, with the numerical values shown in Table 1 , we propose the following conjecture: 
Conjecture 5.3. For ordered trees with $n$-leaves, where $n \geq 3$,

$$
\delta_{\min }(n)=\frac{1}{6}\left\{4 n^{3}-9 n^{2}-13 n+42-3(2 n+3)\left\lfloor\frac{n-1}{2}\right\rfloor+9\left(\left\lfloor\frac{n-1}{2}\right\rfloor\right)^{2}\right\} .
$$

Note that this would imply that the difference between $\delta_{\max }(n)$ and $\delta_{\min }(n)$ for ordered trees grows like $O\left(n^{2}\right)$, although both $\delta_{\max }(n)$ and $\delta_{\min }(n)$ are of $O\left(n^{3}\right)$.

\section{Diameter of $\mathscr{T}_{n}^{\mathrm{o}}$}

In the same spirit as the work done in [1] and in [4] for unrooted trees and plain rooted trees, respectively, we obtain the following result for ordered trees:

Proposition 6.1. Let $\operatorname{diam}_{S P R}\left(\mathscr{T}_{n}^{\circ}\right)$ denote the diameter of $\mathscr{T}_{n}^{\mathrm{o}}$, defined as the maximum value of $d_{S P R}\left(T, T^{\prime}\right)$ over all trees $T, T^{\prime} \in \mathscr{T}_{n}^{\mathrm{o}}$. Then,

$$
\frac{2}{3} n-o(n) \leq \operatorname{diam}_{S P R}\left(\mathscr{T}_{n}^{\mathrm{o}}\right) \leq n-2 .
$$

Proof. Following [1], we can use $\left[\delta_{\max }(n)\right]^{\text {diam }{ }_{S P R}\left(\mathscr{T}_{n}^{\circ}\right)} \geq D(n)$ to obtain the above lower bound for $\operatorname{diam}_{S P R}\left(\mathscr{T}_{n}^{\mathrm{o}}\right)$. Here, $D(n)$ is the number of ordered trees (c.f. (2.1)), whereas $\delta_{\max }(n)$ is given by (5.12). Using Stirling's approximation for the factorial function and carrying out a similar set of steps as in [1], one can show that $\lim _{n \rightarrow \infty} \operatorname{diam}_{S P R}\left(\mathscr{T}_{n}^{\mathrm{o}}\right) / n=$ $2 / 3$ and thus obtain the proposed lower bound. For the upper bound, the proof from [4] for plain rooted trees can be applied to ordered trees as well.

Note. For plain rooted trees, the lower bound on $\operatorname{diam}_{S P R}\left(\mathscr{T}_{n}^{\mathrm{r}}\right)$ obtained using the same approach as above is $n / 2-o(n)$ [4].

Acknowledgments. The author thanks Jotun Hein and Derek Phillips for useful discussion. Two anonymous referees are also thanked for helpful comments on improving the exposition. This research is supported by EPSRC under grant HAMJW and by MRC under grant HAMKA.

\section{References}

1. B.L. Allen and M. Steel, Subtree transfer operations and their induced metrics on evolutionary trees, Ann. Combin. 5 (2001) 1-13.

2. J. Hein, A heuristic method to reconstruct the history of sequences subject to recombination, J. Mol. Evol. 36 (1993) 396-405.

3. E. Schröder, Vier combinatorische probleme, Zeit. für. Math. Phys. 15 (1870) 361-376.

4. Y.S. Song, On the combinatorics of rooted binary phylogenetic trees, Ann. Combin. 7 (2003) 365-379.

5. Y.S. Song and J. Hein, Parsimonious reconstruction of sequence evolution and haplotype blocks: Finding the minimum number of recombination events, In: Algorithms in Bioinformatics (Proceedings of WABI 2003), G. Benson and R. Page, Eds., Springer-Verlag, Berlin, 2003, pp. 287-302.

6. D.L. Swofford and G.J. Olsen, Phylogeny reconstruction, In: Molecular Systematics, D.M. Hillis et al., Eds., Sinauer Associates, Massachusetts, 1990, pp. 411-501. 